\title{
Guía de práctica clínica Ibero-Latinoamericana sobre la esofagitis cáustica en Pediatría: Fisiopatología y diagnóstico clínico-endoscópico (1 ${ }^{\text {a }}$ Parte)
}

\author{
Ibero-latinamerican clinical practical guidelines on pediatric caustic esophagitis: \\ Physiopathology and clinical-endoscopic diagnosis ( $1^{\text {st }}$ Part)
}

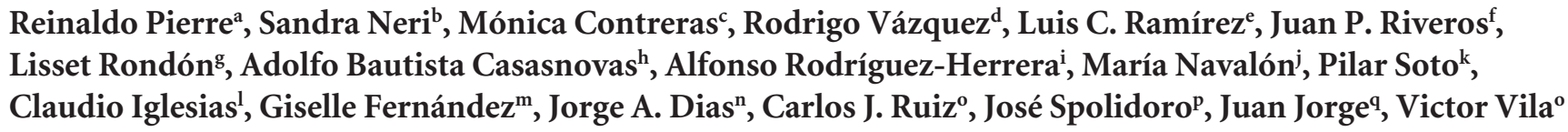

aClínica Razetti de Barquisimeto, Barquisimeto, Venezuela

"Hospital de Niños "Dr. JM de los Ríos", Caracas, Venezuela

"Hospital de Pediatría S.A.M.I.C. "Prof. Dr. Juan P. Garrahan", Buenos Aires, Argentina

dHospital Infantil de México Federico Gómez, México DF, México

eHospital Federico Lleras Acosta, Ibagué, Colombia

'GastriCare Kids, Bogotá, Colombia

Instituto de Ortopedia Infantil Roosevelt, Bogotá, Colombia

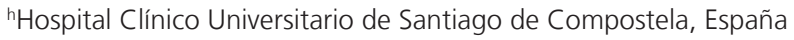

'Grupo IHP, España

¡Hospital Universitario Virgen de la Arrixaca, Murcia, España

kHospital Metropolitano de la Florida, Santiago, Chile

'Hospital Pediátrico, Centro Hospitalario Pereira Rossell, Montevideo, Uruguay

mHospital Infantil Dr. Robert Reid Cabral, Santo Domingo, República Dominicana

${ }^{n}$ Centro Hospitalario S. Joao, Porto, Portugal

- Hospital Sant Joan de Deu, Barcelona, España

pNúcleo de Formación em Pediatria da Escola de Medicina da PUCRS, Porto Alegre, Brasil

${ }^{\natural}$ Centro Gastrodiagnóstico y Especialidades, Santo Domingo, República Dominicana

Recibido: 11 de junio de 2019; Aceptado: 3 de octubre de 2019

¿Qué se sabe del tema que trata este estudio?

La ingesta accidental de cáusticos en Pediatría y sus complicaciones representan un problema de salud pública en América Latina. A pesar de lo relevante del tema, existen áreas controversiales en relación al manejo de esta patología , y una ausencia de evidencia científica dado la escasa cantidad de estudios experimentales, controlados que permitan establecer recomendaciones.
¿Qué aporta este estudio a lo ya conocido?

Se trata de la Primera Guía de Práctica Clínica Ibero-Latinoamericana sobre la Esofagitis cáustica en Pediatría, basada en la revisión de la literatura y en un análisis de la evidencia científica disponible de acuerdo a la metodología Grade. El presente manuscrito pretende ofrecer al clínico una serie de recomendaciones con el fin de orientar una practica clínica más segura y efectiva basada en la evidencia científica para estos pacientes.

Correspondencia:

Reinaldo Pierre

vice.presidencia.laspghan@gmail.com 


\section{Resumen}

La ingestión de cáusticos representa un grave problema médico-social por las consecuencias devastadoras e irreversibles que puede producir en el tracto digestivo superior. En Iberoamérica no se han publicado datos fidedignos sobre la incidencia o la prevalencia de lesiones inducidas por cáusticos. La información disponible sobre la presentación clínica, diagnóstico, tratamiento y pronóstico se basa en series retrospectivas de casos y, de hecho, su manejo clínico se sustenta en muchos casos fundamentalmente en la opinión de expertos. Recientemente como una iniciativa de la Sociedad Latinoamericana de Gastroenterología, Hepatología y Nutrición Pediátrica (SLAGHNP) y con la colaboración de colegas de la Sociedad Española de Gastroenterología, Hepatología y Nutrición Pediátrica (SEGHNP), hemos diseñado una Guía de Práctica Clínica (GPC) la cual incluye una serie de enunciados y recomendaciones dirigidos a optimizar la atención a los pacientes y que se basan en la revisión sistemática de la evidencia. En dos (2) manuscritos sucesivos nos hemos enfocado primero, en los aspectos fisiopatológicos y de diagnóstico clínico-endoscópico de la esofagitis cáustica en niños ( $1^{\text {a }}$. Parte) y en segundo lugar, en los aspectos más relevantes del tratamiento (2a. Parte). Esperamos esta guía se convierta en una herramienta útil para el clínico en el difícil proceso de toma de decisiones a la hora de evaluar un paciente posterior a la ingesta de una sustancia cáustica.

\section{Abstract}

Caustic ingestion represents a serious social-medical problem due to the devastating and irreversible consequences it can produce in the upper digestive tract. In Ibero-America, there are no published reliable data on the incidence or prevalence of caustic-induced injuries, and most of the available information on clinical presentation, diagnosis, treatment, and prognosis is based on retrospective clinical series and, indeed, its clinical management is often based primarily on expert opinion. Recently as an initiative of the Latin American Society for Pediatric Gastroenterology, Hepatology and Nutrition (LASPGHAN) and with the cooperation of the Spanish Society for Pediatric Gastroenterology, Hepatology and Nutrition (SEGHNP), we have designed a Clinical Practice Guideline that include a series of statements and recommendations aimed at optimizing patient medical care which is based on the systematic review of evidence. Two (2) successive papers focused on the evaluation of physiopathological and clinical-endoscopic diagnostic features of caustic esophagitis in children ( $1^{\text {st }}$. Paper) and, on the other hand, the most relevant therapeutic considerations ( $2^{\text {nd }}$. Paper $)$. We expect this guideline to become a useful tool for the physician in the difficult decision-making process when assessing patients after caustic ingestion.
Palabras clave:

esofagitis;

cáustico;

guía de práctica clínica; diagnóstico;

tratamiento

\section{Keywords:}

esophagitis; caustic; clinical practice guideline; diagnosis; treatment

\section{Introducción}

La ingestión de cáusticos en niños constituye un problema de salud pública, especialmente en los países en vías de desarrollo, y un importante problema médico-social debido a las consecuencias devastadoras e irreversibles sobre la integridad física del niño. Se calcula una incidencia de 38,7 casos/100.000 habitantes, con una mortalidad del 1-4\% en los países occidentales $^{1,2}$. A. Bautista y F. Arguelles realizaron una revisión bibliográfica de datos epidemiológicos de España y otros países. Así, en Francia, alcanza hasta el $4,5 \%$ del total de ingresados por accidentes. En áreas del norte de Europa, la frecuencia anual alcanza cifras de 5/100.000 pacientes menores de 5 años. En Estados Unidos, anualmente se producen más de 5000 casos de ingestión de sustancias químicas cáusticas; de éstos, el 50-80\% ocurren en la población pediátrica (meno- res de 18 años) $)^{3}$. En Iberoamérica no se han publicado datos fidedignos sobre la incidencia, prevalencia o mortalidad de las lesiones inducidas por cáusticos. La información disponible sobre la presentación clínica, diagnóstico, tratamiento y pronóstico se basa generalmente en series retrospectivas de casos y estudios no controlados. Es por esta razón que como una iniciativa de las Sociedades Latinoamericana (SLAGHNP) y Española (SEGHNP) de Gastroenterología, Hepatología y Nutrición Pediátrica se formó en el año 2018 un Grupo de Trabajo con el objetivo de elaborar una guía de práctica clínica (GPC), la cual contiene una serie de enunciados relacionados con fisiopatología, clínica, diagnóstico y tratamiento de la esofagitis cáustica en Pediatría (pacientes menores de 18 años) y para cada uno de los cuales se ha desarrollado un argumento explicativo que conlleva a una serie de recomendaciones. El objetivo del presente trabajo es establecer recomen- 
daciones que puedan orientar al clínico en el difícil proceso de toma de decisiones a la hora de evaluar un paciente posterior a la ingesta de una sustancia cáustica.

\section{Métodos}

Tomando como modelo el concepto de Medicina Basada en Evidencia (MBE) hemos decidido a través del presente manuscrito realizar un análisis de la evidencia disponible desde la perspectiva de los diferentes escenarios clínicos y establecer grados de recomendación para el ejercicio de procedimientos diagnósticos y terapéuticos. En nuestro caso particular y una vez establecidos los enunciados que formarían parte de la guía, decidimos trabajar con el sistema Grading of Recommendations, Assessment, Development and Evaluation $(\text { GRADE })^{4}$, el cual se caracteriza por valorar la evidencia según el área temática o escenario clínico y el tipo de estudio que involucra al problema clínico en cuestión. En el sistema GRADE la calidad de la evidencia se clasifica, inicialmente, en alta o baja, según provenga de estudios experimentales u observacionales, posteriormente, según una serie de consideraciones, el nivel de evidencia queda en alto, moderado, bajo o muy bajo.

La fuerza de las recomendaciones puede ser fuerte o débil y se apoya no sólo en la calidad de la evidencia, sino en una serie de factores tales como el balance entre riesgo y beneficio, los valores y preferencias de pacientes y profesionales y el consumo de recursos o costos ${ }^{4}$.

Se revisaron las principales publicaciones científicas clasificándolas de acuerdo a diseño experimental (estudios experimentales, descriptivos, de cohorte, revisiones sistemáticas, meta-análisis) y de acuerdo a subtemas específicos (fisiopatología, clínica, diagnóstico, tratamiento, etc.). Se dió preferencia a artículos publicados en los últimos 5 años en idioma español, portugués o inglés y a aquellos de mayor nivel de evidencia. En el caso de publicaciones de mucha rigurosidad estadística y evidencia de alta calidad se decidió incluir trabajos de mayor antigüedad. Los buscadores utilizados fueron Bireme, MedLine, Pubmed, Lilacs, Scielo y la Red Cochrane.

La búsqueda se hizo mediante el procedimiento de palabras clave (esofagitis, cáustica, diseño experimental, niños).

En dos (2) manuscritos sucesivos hemos evaluado en primer lugar, aspectos de la fisiopatología y el diagnóstico clínico-endoscópico de la esofagitis cáustica en niños, y en segundo lugar, todo lo relacionado con el tratamiento. Los presentes manuscritos fueron evaluados y aprobados por los miembros del Grupo de Trabajo y por un grupo de especialistas en el área que actuaron como revisores externos.

\section{Epidemiología}

Desde el punto de vista epidemiológico el grupo de edad de mayor riesgo son los menores de 5 años con un pico de incidencia alrededor de los 2 años. Recomendamos en todos los casos investigar si la ingesta fue voluntaria o accidental. Recomendación débil, nivel de evidencia bajo

Según las estadísticas la ingesta de cáusticos en adolescentes y adultos corresponde generalmente a intentos suicidas y por lo tanto es de carácter voluntario, no-accidental ${ }^{1,2}$. La intencionalidad en estos casos se asocia a un mayor volumen de sustancia ingerida y a una mayor severidad de las lesiones ${ }^{3}$. En la población infantil la ingesta de cáusticos por el contrario es casi siempre accidental lo cual podría suponer en teoría un riesgo menor de lesión.

En cuanto al pronóstico en relación al lugar de procedencia, no hay muchos estudios realizados ni amplias diferencias según los reportes clínicos de las lesiones cáusticas en la población rural vs. la población urbana. La tendencia pareciera ser a lesiones más graves y un peor pronóstico en la población rural, sobre todo por el retardo en la atención médica especializada que generalmente se concentra en los grandes centros urbanos $^{2}$.

\section{Fisiopatología}

Las sustancias cáusticas tienen propiedades diferentes y particulares de acuerdo a su pH (ácidos $v s$ álcalis) y la fisiopatología es diferente en cada caso. Recomendamos la identificación del tipo de cáustico de manera rutinaria en todos los casos. Recomendación fuerte, nivel de evidencia moderado

Los álcalis destruyen lípidos y proteínas, alterando la arquitectura celular, induciendo necrosis por licuefacción ${ }^{1-3,6,7}$. La saponificación de lípidos permite la penetración profunda a la submucosa y muscular, lo cual puede resultar en trombosis vascular, fibrosis e inclusive perforación ${ }^{4-7}$. El ácido del estómago suele neutralizar el álcali. Los álcalis con $\mathrm{pH} \leq 12$ pocas veces causan lesiones, a menos que estén a gran concentración; sin embargo, un agente con $\mathrm{pH}>12$ causa lesiones importantes, independientemente de la concentración ${ }^{7,9}$. El blanqueador doméstico es el principal agente cáustico ingerido; tradicionalmente contiene hipoclorito de sodio, una solución alcalina con un $\mathrm{pH} \geq 11$ que usualmente se presenta a una concentración variable entre 5 y 12\%, siendo menos peligrosa la de menor concentración $(7,9,10)$. Los ácidos constituyen entre el 3\%-40\% de las ingestas de cáusticos, dependiendo de la zona geográfica ${ }^{8,10-13}$. La ingesta de ácidos fuertes con 
$\mathrm{pH}<2$ resulta en necrosis coagulativa como resultado de la isquemia con menor probabilidad de penetrar y perforar $^{13-18}$.

Desde el punto de vista fisiopatológico se reconocen al menos 4 fases en la evolución del proceso inflamatorio post-exposición al cáustico. Se recomienda individualizar el manejo y correlacionar algunas decisiones diagnósticas con el tiempo de evolución. Recomendación fuerte, nivel de evidencia moderado

La severidad de las lesiones por cáusticos depende de múltiples factores, siendo el tipo de cáustico $(\mathrm{pH})$, el volumen ingerido, la intencionalidad y el tiempo de contacto con el tubo digestivo algunos de los más importantes en la fisiopatología del daño ${ }^{19}$. El riesgo de desarrollo de estenosis es directamente proporcional a la severidad de la lesión inicial ${ }^{20,21}$.

En 2004, un estudio de Poley et al. demostró que las lesiones por ácidos fuertes tienen rápida penetración en los tejidos y generan daño de la pared esofágica ${ }^{5}$.

El tiempo de contacto con la mucosa del tubo digestivo dependerá además del estado del cáustico, ya sea sólido o líquido. En estudios animales se demostró además que la concentración de la solución es el predictor más importante de daño epitelial ${ }^{22-24}$.

Desde el punto de vista fisiopatológico la lesión por cáusticos se produce en 4 fases ${ }^{14,15}$ :

Fase 1 (día 1 a 3): desde las primeras horas, se produce una reacción inflamatoria con edema y necrosis eosinofílica. En las horas siguientes a la ingesta se producen en la mucosa especies reactivas de oxígeno y radicales libres que pueden mantenerse elevados hasta 72 horas post injuria contribuyendo al daño inicial y al desarrollo de estenosis secundarias ${ }^{26}$.

Fase 2 (día 3 a 5): ulceración, puede producirse perforación si la úlcera compromete la capa muscular.

Fase 3 (día 6 a 14): principalmente desde el día 10 comienzan los fenómenos reparatorios y la aparición de tejido de granulación.

Fase 4 (día 15 a 30): fase de cicatrización, con reepitelización de la mucosa ulcerada. Se establece la estenosis esofágica ${ }^{27-29}$.

\section{Clínica}

La clínica es enormemente variable y puede ir desde ningún síntoma o molestias orofaríngeas leves hasta síntomas más severos. Esta gran variabilidad clínica sugiere la necesidad de una observación, evaluación y seguimiento rigurosos en cada caso. Recomendación fuerte, nivel de evidencia bajo
Las manifestaciones clínicas iniciales después de la ingestión de un cáustico pueden incluir disfagia, sialorrea, dolor abdominal y hematemesis. La relación entre la gravedad de los síntomas a nivel orofaríngeo y la severidad de las lesiones esofágicas sigue siendo incierta (figura 1) ) $^{30,31,34,35,36}$. Gorman y cols. en un estudio observacional de tipo prospectivo encontraron que síntomas tales como vómitos, sialorrea y estridor eran predictivos de una lesión esofágica grave, definida como quemadura esofágica de segundo grado o más, a diferencia de los pacientes que no presentaban signos o solo uno de ellos, los cuales tuvieron endoscopias con hallazgos negativos ${ }^{35}$. El análisis que realiza Gorman en su publicación es que como signo único, las quemaduras orales tienen solo una sensibilidad del 83 $\%$ para detectar quemaduras esofágicas, un valor predictivo positivo de sólo el $31 \%$ y un valor predictivo negativo relativamente bajo de $79 \%$. En pacientes con quemaduras orales, la decisión de realizar una endoscopia debe basarse tanto en la intención de la ingestión así como en la evaluación de otros signos y síntomas clínicos $^{35}$. Al intentar predecir una lesión esofágica, el grupo de síntomas clínicamente más útiles son: vómitos, disfagia, dolor abdominal y la presencia de quemaduras orales. Si dos o más de estos signos y síntomas están presentes, la probabilidad de encontrar lesiones esofágicas es más elevada (sensibilidad $=94 \%$, valor predictivo positivo $=43 \% \mathrm{y}$ valor predictivo negativo $=96 \%)^{35,36}$.

Betalli et al. realizaron un estudio observacional multicéntrico que incluyo 162 pacientes, encontrando que la presencia de síntomas fue el factor de predicción más potente de lesiones esofágicas graves ${ }^{28}$. Durante el estudio se realizó una clasificación de signos y

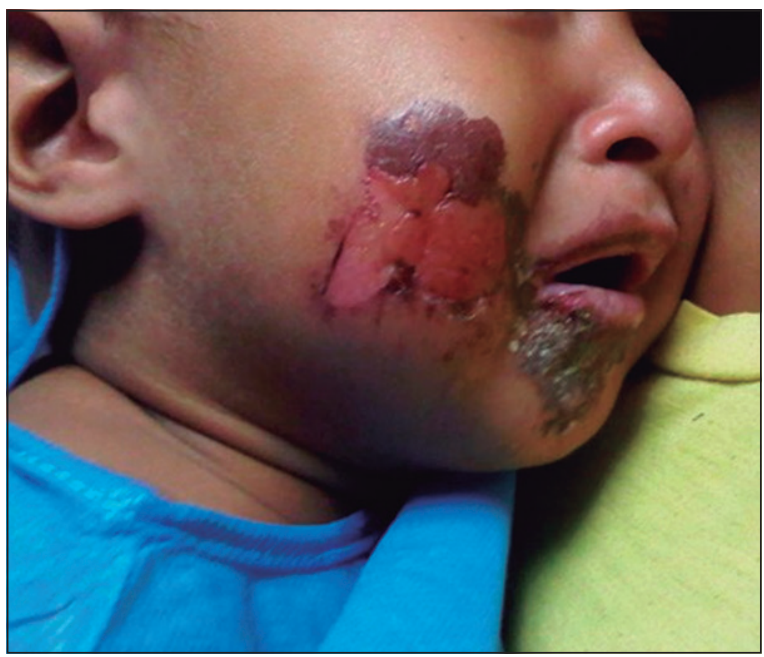

Figura 1. Lesiones en mucosa oral y región facial en un niño posterior a la ingesta de cáustico (Imagen cortesía Dra. María Teresa Artís, Venezuela). 
síntomas, considerándose síntomas menores: lesiones bucales y/o bucofaríngeas y vómitos; y síntomas mayores: disnea, disfagia, sialorrea y hematemesis. Teniendo en cuenta estos criterios, se identificaron lesiones esofágicas leves en 143 de 162 pacientes $(88,3 \%)$ y lesiones esofágicas graves (tercer grado) en 19 pacientes $(11,7 \%)$.

$\mathrm{El}$ riesgo de lesiones esofágicas graves en pacientes sin signos y/o síntomas fue muy bajo (odds ratio [OR] 0,13 [IC $95 \%, 0,02-0,62$ ], P Z 0,002); mientras que la presencia de 3 o más síntomas se comportó como un importante predictor de lesión esofágica grave (OR 11,97 [IC $95 \%, 3,49-42,04]$, P Z 0,0001) $)^{28,37}$.

En pacientes pediátricos la ausencia de síntomas posterior a la ingesta de una sustancia cáustica puede ser un indicador de ausencia de lesiones o presencia de lesiones de bajo grado. La realización de la endoscopia puede ser determinada en base a criterios clínicos. Recomendación fuerte, nivel de evidencia alto

Aunque la correlación entre la falta de síntomas y la ausencia de lesiones no se puede aplicar a los pacientes que ingieren intencionalmente una sustancia cáustica, la mayoría de los estudios realizados en niños en los cuales la ingesta es casi siempre accidental, muestran que la ausencia de hallazgos clínicos puede ser un indicador de ausencia de lesiones o presencia de lesiones de bajo grado.

En una revisión reciente de las guías de la Sociedad Europea de Endoscopia Gastrointestinal y la Sociedad Europea de Gastroenterología, Hepatología y Nutrición Pediátrica (ESGE- ESPGHAN, por sus siglas en inglés), el panel de expertos sugiere que cada niño que haya ingerido una sustancia corrosiva debe someterse a un seguimiento exhaustivo y la realización de una endoscopia puede ser determinada en base a la presencia de síntomas $^{39}$. Ante un niño con sospecha de ingestión cáustica, sintomático (cualquier tipo de lesión orofaríngea, sialorrea, disfagia, hematemesis, disnea, dolor abdominal, etc.) debe practicarse una esófago-gastroduodenoscopia (EGD) para poder identificar o descartar lesiones a nivel del tracto digestivo (Recomendación fuerte, nivel de evidencia alto).En el caso de sospecha de ingestión de corrosivo el estudio endoscópico puede ser diferido si el niño se encuentra asintomático y se garantiza un seguimiento clínico adecuado (Recomendación débil, nivel de evidencia moderado ${ }^{39}$.

En una editorial del Journal of Pediatric Gastroenterology and Nutrition, Kay y Wyllie afirman que la literatura publicada sobre el manejo de la ingestión de sustancias cáusticas es aún limitada y confusa. Recomiendan no realizar estudio endoscópico en pacientes con sospecha de ingestión cáustica que se encuentren asintomáticos y sin quemaduras orofaríngeas ${ }^{38,41}$.

\section{Diagnóstico}

Los niños que hayan ingerido una sustancia corrosiva deben ser valorados exhaustivamente. La presencia de síntomas determinará la necesidad de realizar una endoscopia y por lo tanto de trasladar al paciente si el centro de asistencial no dispone de ese recurso. Cuando el paciente presenta una completa ausencia de signos y síntomas, y tratándose de una ingesta no intencional, no estaría indicada la endoscopia ${ }^{39}(R e-$ comendación fuerte, nivel de evidencia moderado). La ausencia de síntomas tiene un valor predictivo de ausencia de lesiones severas en el esófago de 0,03, lo que otorga al niño asintomático un riesgo relativo de lesiones muy bajo (OR 0,13 [95\% CI, 0,02-0,62], P $0,002)^{28}$.

Los niños que presenten síntomas mayores (sialorrea, disnea, disfagia y hematemesis) son los que tienen mayor riesgo de daño anatómico esofágico (OR 8,98 [95\% CI, 2,75-31,07], P Z 0,0001. Entre los síntomas mayores, los niños que se presentan con disnea tendrán el mayor riesgo de tener lesiones anatómicas severas (OR 16.71 [95\% CI, 1,1-493,8], P Z 0,04) ${ }^{28}$.

Los niños con síntomas menores (vómitos, lesiones bucales, etc.) suelen tener menor riesgo de daño esofágico que los anteriores (OR 2,92 [95\% CI, 0,92-9,86], $\mathrm{P} \mathrm{Z} 0,05)^{28}$.

En los pacientes con ingesta voluntaria, no-accidental, aunque estén asintomáticos, se recomienda siempre realizar una endoscopia (Recomendación fuerte, nivel de evidencia bajo ${ }^{42}$.

La evaluación pre-endoscópica puede incluir la utilización de escalas de valoración clínica que permiten predecir el riesgo de lesión y estenosis cáustica (p. ej. escala de Drool). Recomendación débil, nivel de evidencia bajo

En medicina de emergencia, es muy importante determinar los criterios de seguridad para dar de alta a los pacientes sin necesidad de practicar más exploraciones. El uso de sistemas de puntuación es una forma de implementar un algoritmo de decisión que permita los mejores resultados para el paciente junto al uso más eficiente de recursos ${ }^{40,43}$.

Una herramienta útil para la toma de decisiones es el empleo de la escala Drool, la cual se desarrolló a partir de la observación de que la presencia de algunos síntomas permite pronosticar el desarrollo de estenosis en caso de quemaduras esofágicas (OR 11,97 [95\% CI, 3,49-42,04] P Z 0,0001) $)^{28,40}$.

Considerando este hecho se elaboró la escala de puntuación Drool tras el análisis de los hallazgos en una serie de 202 que ingirieron cáusticos y presentaron al menos un síntoma. A estos niños se les realizó 
Tabla 1. Escala de Drool para ingestión de cáusticos

\begin{tabular}{|c|c|c|c|c|}
\hline Componente del acrónimo & Signos y síntomas & 0 Puntos & 1 Punto & 2 puntos \\
\hline Descarga salivar (Drooling) & Descarga de saliva, babeo & $\geq 12$ horas & $<12$ horas & No \\
\hline Rechazo al alimento (Reluctance) & $\begin{array}{l}\text { Rechazo a comer, disfagia o falta de } \\
\text { tolerancia oral }\end{array}$ & $\geq 24$ horas & $<24$ horas & No \\
\hline Orofaringe (Oropharynx) & Quemaduras orales u oro-faríngeas & $\begin{array}{l}\text { Lesiones severas como friabi- } \\
\text { lidad, hemorragia, vesicular, } \\
\text { membranas blanquecinas, } \\
\text { necrosis o exudados }\end{array}$ & $\begin{array}{l}\text { Edema o } \\
\text { hiperemia }\end{array}$ & No \\
\hline Otros (Others) & $\begin{array}{l}\text { Número total de otros síntomas: fiebre } \\
\text { persistente, hematemesis, malestar } \\
\text { abdominal, dolor retroesternal o disnea }\end{array}$ & $\geq 2$ síntomas & 1 síntoma & No \\
\hline Leucocitosis & Leucocitosis & $\geq 20.000$ & $<20.000$ & No \\
\hline
\end{tabular}

una endoscopia en las 48 horas siguientes al evento. Se excluyeron intencionalmente los niños que no presentaron síntomas. Una puntuación igual o inferior a 4 puntos se asoció a una alta probabilidad de desarrollo de lesiones estenosantes esofágicas (valor predictivo positivo de $85 \%)^{40}$. La escala se presenta en la tabla 1 .

El uso de la escala Drool permite predecir el riesgo de desarrollo de estenosis esofágica y el uso adecuado de medidas de prevención de estenosis en aquellos pacientes que lo requieran.

La recomendación es la realización de la endoscopia durante las primeras $\mathbf{1 2 - 4 8}$ horas posteriores a la ingesta. No se recomienda la endoscopia después de la primera semana debido al alto riesgo de perforación. Recomendación fuerte, nivel de evidencia bajo

El momento ideal para realizar la exploración endoscópica tras una ingesta de cáusticos en niños es controvertido $^{16}$. La mayoría de estudios coinciden en recomendar su realización en las primeras 24 horas post-ingesta para evaluar la extensión de las lesiones, establecer el pronóstico y guiar el tratamiento ${ }^{28,44}$.

Son numerosos los estudios que recomiendan la endoscopia "precoz"; se trata en su mayoría de estudios retrospectivos o prospectivos observacionales ${ }^{45-47}$ $y$ en otros casos de revisiones ${ }^{8,20,49,50}$.

Esta recomendación coincide con los documentos de consenso de la European Society of Gastrointestinal Endoscopy (ESGE), de la European Society for Paediatric Gastroenterology Hepatology and Nutrition (ESPGHAN $)^{39}$ y de la American Society for Gastrointestinal Endoscopy (ASGE) ${ }^{51}$.

Sin embargo, hay autores que en esta recomendación de endoscopia "precoz" amplían el período de exploración endoscópica hasta 48 horas postingesta ${ }^{1} y$ otros que afirman que la exploración endoscópica es segura hasta 4 días después ${ }^{5,44,45}$.

La endoscopia tras 4 días post-ingesta ("tardía”) parece aumentar el riesgo de perforación, por lo que no se recomienda ${ }^{45}$. De hecho, existe escasa evidencia en torno a la seguridad de la endoscopia realizada tras 5-15 días post-ingesta ${ }^{45,51}$.

Existe controversia en cuanto a la realización de endoscopia en pacientes sin signos o síntomas de lesión cáustica (pacientes de bajo riesgo). Sin embargo y según la evidencia más consistente, actualmente no recomendamos la realización de endoscopia en el paciente pediátrico de "bajo riesgo". Recomendación débil, nivel de evidencia moderado

Estudios publicados en los últimos años sugieren la necesidad de practicar endoscopia sólo en pacientes con alto riesgo de lesiones digestivas. En cuanto a los pacientes asintomáticos y sin signos (pacientes de "bajo riesgo") la evidencia más reciente sugiere la necesidad de un "enfoque mínimamente invasivo" ${ }^{0,51}$. En el caso de pacientes pediátricos con ingesta involuntaria y sin síntomas, se recomienda establecer un periodo de observación para mayor seguridad y corroborar que siguen asintomáticos tras la introducción de la dieta oral $^{34}$.

En esta misma línea, varios estudios recomiendan no realizar endoscopia de rutina a los pacientes pediátricos de "bajo riesgo", sino seguimiento clínico y actitud expectante ${ }^{27,28}$. Otros argumentan que la ausencia de hallazgos clínicos en el niño se correlacionó en la mayor parte de casos con la ausencia de lesiones esofágicas o con lesiones de bajo grado y la exploración endoscópica no cambió la actitud terapéutica del clínico, contribuyendo además a un aumento del gasto sanitario y a un aumento del riesgo de iatrogenia para el paciente. Sin embargo, en contra de esta recomendación existen 3 estudios retrospectivos en los que los autores encontraron lesiones esofágicas significativas en una proporción de pacientes asintomáticos $(26 \%$, $19 \%$ y $12 \%$, respectivamente) por lo que estos autores 

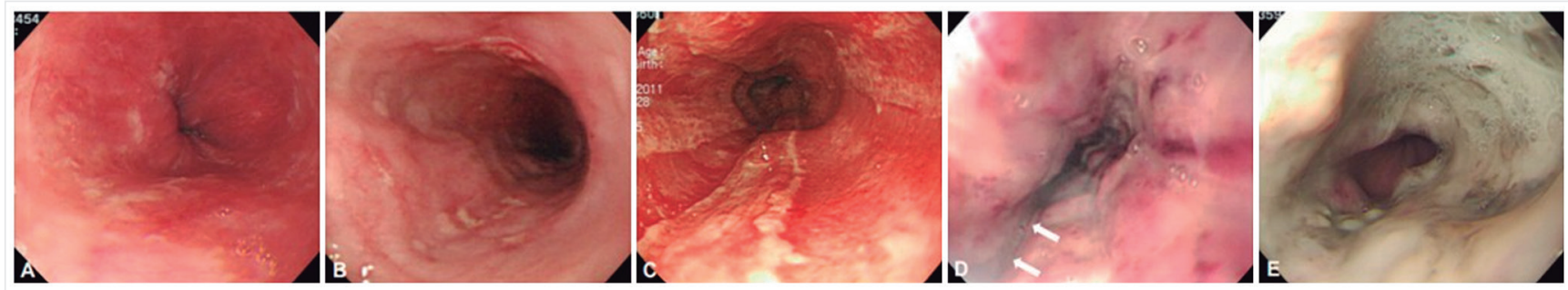

Figura 2. Clasificación de Zargar (Grados 1-3B). Fuente: Park KS. Evaluation and management of caustic injuries from ingestion of acid or alcaline substances. Clin Endosc 2014;47(4):301-7. Rossi A. Acute caustic ingestion: State of the art and new trends. Journal of GHR (Internet) 2015 March 21 (citado 07 Ene 2017);4(3):1501-6.

consideran mandatoria la realización de endoscopia ante toda ingesta de caústicos ${ }^{18}$.

En conclusión, según el análisis de la evidencia, actualmente no se recomienda la realización de endoscopia en el paciente pediátrico de "bajo riesgo" (sin signos ni síntomas), con un grado de recomendación débil.

El diagnóstico endoscópico y establecer el grado de severidad de las lesiones es fundamental ya que orienta la toma de decisiones y permite establecer la conducta terapéutica y el pronóstico. Se recomienda utilizar la clasificación de Esofagitis cáustica de Zargar. Recomendación Fuerte, nivel de evidencia bajo

La endoscopia digestiva alta se puede utilizar para evaluar lesiones esofágicas y gástricas, establecer el pronóstico y el plan de manejo ${ }^{28}$, sin embargo, está contraindicada en pacientes hemodinámicamente inestables, con sospecha de perforación, que presenten dificultad respiratoria severa y pacientes con edema laríngeofaríngeo grave.

El sistema de clasificación para esofagitis cáustica de Zargar ayuda a establecer la extensión y profundidad de las lesiones, las alteraciones motoras, predecir los resultados clínicos y definir el pronóstico ${ }^{45}$ (figura 2).

Esta clasificación comprende cuatro (4) grados de severidad:

Grado 0: Mucosa normal; Grado 1: Ligero edema y enrojecimiento de la mucosa; Grado 2A: Úlceras superficiales, hemorragias y exudados; Grado 2B: Ulceración profunda local o circundante; Grado 3A: Necrosis focal; Grado 3B: Necrosis extensa; Grado 4: Perforación. La mayoría de los pacientes con lesiones de grado $1 \mathrm{o} 2 \mathrm{~A}$ tienen un buen pronóstico sin riesgo de muerte o de desarrollo de complicaciones inmediatas o tardías. Para los pacientes con lesiones de grado 3B la mortalidad puede ser hasta del 65\% y, en la mayoría de los casos, es necesario la resolución quirúrgica $^{45,51,52}$.
Además de la endoscopia, otros estudios tales como radiología, tomografía, etc. pueden jugar un papel en la evaluación del paciente con esofagitis cáustica. La utilización de estos métodos no se recomienda de rutina y depende de factores clínicos específicos. Recomendación débil, nivel de evidencia bajo

El uso de la radiografía simple toraco-abdominal, se ha pregonado frente a la sospecha de perforación, buscando signos como la presencia de aire en el mediastino o infradiafragmático. De igual forma, los estudios contrastados buscando fugas de contraste y la tomografía computada buscando sombras que revelen presencia de aire mediastinal o subdiafragmático, pueden realizarse previo a la endoscopia digestiva o en el transcurso del tratamiento con dilataciones, si existe la sospecha clínica de perforación ${ }^{8,16,49}$.

Millar y col. han propuesto el uso de un estudio de medicina nuclear para la detección de lesiones cáusticas mezclando un radioisótopo tipo Tecnecio-99 con sucralfato. Un scan posterior demuestra que en las lesiones grado 1 y $2 \mathrm{~A}$ no hay retención del fármaco en el esófago, mientras que en las lesiones grado $2 \mathrm{~B}$ ó superiores es posible demostrar la retención del radiofármaco. La sensibilidad del método fue del $100 \%$ y la especificidad del $81 \%$, con un valor predictivo positivo del $84 \%$ y un valor predictivo negativo del $100 \%{ }^{53}$.

El uso del ultrasonido endoscópico (USE) ha sido propuesto para la evaluación adicional de las lesiones esofágicas por ingesta de cáusticos. Los estudios han sido pocos y realizados básicamente en adultos y si bien han mostrado ser efectivos en reflejar la destrucción de la capa muscular con cierto valor pronóstico, no ha demostrado ser superior que la endoscopia convencional para predecir complicaciones tempranas o tardías ${ }^{54,55}$.

La Tomografía Computarizada (TC) también ha sido propuesta como predictor de estenosis esofágica. Los estudios son escasos, realizados en adultos, no son prospectivos ni randomizados. En general, si bien la TC es un estudio muy prometedor, no ha demostrado aún ser superior a la Endoscopia ${ }^{56,57}$. 


\section{Tabla 2. Resumen. Enunciados, Recomendaciones y Niveles de evidencia}

Enunciados

\section{Manifestaciones clínicas}

La gran variabilidad clínica sugiere la necesidad de una observación, evaluación y seguimiento rigurosos en cada caso

La realización de la endoscopia debe ser determinada en base a criterios clínicos. No se recomienda de rutina una endoscopia si la ingesta ha sido accidental y el paciente está con ausencia de síntomas orofaríngeos y/o sistémicos

Se recomienda realizar una endoscopia alta en pacientes con ingestión intencional así estén asintomáticos

\section{Diagnóstico}

Se recomienda utilizar la escala DROOL en la evaluación pre-endoscópica para predecir el riesgo de lesión y estenosis

Se sugiere realizar la endoscopia en las primeras 12-48 horas después de la ingesta y no hacerla después de la 1ra. semana debido al alto riesgo de perforación

Para realizar el diagnóstico endoscópico y establecer pronóstico se debe usar la escala de clasificación de esofagitis cáustica de Zargar

La evaluación radiológica (Rx simple, estudios contrastados, tomografía, medicina nuclear) solo debe usarse según factores clínicos específicos y no deben realizarse de rutina
Recomendación

Nivel de evidencia

Recomendación fuerte Nivel de evidencia bajo

Recomendación fuerte Nivel de evidencia moderado

Recomendación fuerte Nivel de evidencia bajo

Recomendación débil Nivel de evidencia bajo

Recomendación fuerte Nivel de evidencia bajo

Recomendación fuerte Nivel de evidencia bajo

Recomendación débil Nivel de evidencia bajo

\section{Conclusiones}

La ingesta de sustancias cáusticas en niños es frecuentemente de carácter accidental e implica un alto riesgo de lesiones potencialmente graves a nivel del tracto digestivo superior. Una interpretación cuidadosa de los hallazgos clínicos, así como un seguimiento cuidadoso son importantes. La endoscopia es una herramienta fundamental que permite no sólo el diagnóstico sino también la elaboración de planes de tra- tamiento específicos. Estudios clínicos randomizados, controlados son necesarios para una mejor definición de estrategias diagnósticas y terapéuticas. Un resumen con los principales enunciados, recomendaciones y los niveles de evidencia que las sostienen se presenta en la tabla 2.

\section{Conflicto de intereses}

Los autores declaran no tener conflicto de intereses.

\section{Referencias}

1. Riffat F, Cheng A. Pediatric caustic ingestion: 50 consecutive cases and a review of the literatura. Diseases of the esophagus. 2009;22 89-94.

2. Christensen H. Epidemiology and prevention of caustic ingestion. Acta Paediatrica 1994;83:212.

3. Bautista Casasnovas A, Argüelles Martin F. Capítulo 14: Ingesta de cáusticos. En Protocolos diagnóstico-terapéuticos de Gastroenterología, hepatología y nutrición pediátrica. Colección: Protocolos de la Asociación Española de Pediatría, págs. 121-130, 2a Edición. Editorial Ergón S.A, Madrid, 2010.

4. Aguayo-Albasini JL, Flores-Pastor B, Soria-Aledo V. Sistema GRADE: clasificación de la calidad de la evidencia y graduación de la fuerza de la recomendación. Cir Esp. 2014;92(2):82-8.
5. Poley J, Steyerberg EW, Kuipers EJ, Dees J, Hartmans R, Tilenus HW, Siersema P. Ingestion of acid and alkaline agents: outcome and prognostic value of early upper endoscopy. Gastrointest endosc. 2004;60:372-7.

6. Arévalo-Silva C, Eliashar R, Wholglertnter J, Eliden J, Gross M. Ingestion of caustic substances: a 15 year experience. Lanryngoscope 2006;116(8):1422-6.

7. Kurowski JA, Kay M. Caustic Ingestions and Foreign Bodies Ingestions in Pediatric Patients. Pediatr Clin N Am 2017;64:50724.

8. Park KS. Evaluation and Management of Caustic Injuries from Ingestion of Acid or Alkaline Substances. Clin Endosc 2014;47(4):301-7.

9. Atug O, Dobrucali A, Orlando RC. Critical pH level of lye ( $\mathrm{NaOH})$ for esophageal injury. Dig Dis Sci 2009;54:980-7.
10. Campos AMS, Bucaretchi F, RodriguesFernandes LC, et al. Toxic Exposures In Children Involving Legally And Illegally Commercialized Household Sanitizers. Rev Paul Pediatr 2017;35:11-7.

11. Rafeey M, Ghojazadeh M, Sheikhi S, et al. Caustic Ingestion in Children: a Systematic Review and Meta-Analysis. J Caring Sci 2016;5(3):251-65.

12. Rafeey M, Ghojazadeh M, Mehdizadeh A, et al. Intercontinental comparision of Caustic Ingestion in Children. Korean J Pediatr 2015;58:491-500.

13. Vandenplas Y. Management of Benign Esophageal Strictures in Children. Pediatr Gastroenterol Hepatol Nutr 2017;20:2115.

14. Contini S, Scarpignato C. Caustic injury of the upper gastrointestinal tract: a comprehensive review. World J Gastroenterol 2013;19:3918-30.

15. Arnold M, Numanoglu A. Caustic 
ingestion in children-a review. Semin Pediatr Surg 2017;26:95-104.

16. Millar AJ, Cox SG. Caustic injury of the oesophagus. Pediatr Surg Int 2015;31:11121.

17. Karaman I, Koc O, Karaman A, et al. Evaluation of 968 children with corrosive substance ingestion. Indian J Crit Care Med 2015;19:714-8.

18. Temiz A, Oguzkurt P, Ezer SS, et al. Predictability of outcome of caustic ingestion by esophagogastroduodenoscopy in children. World J Gastroenterol 2012;18:1098-103.

19. Serizawa M, Rincón E, Morales D, Hernández G. La Ingestión de cáusticos en el niño, un problema de salud pública. Arch Ven Puer Ped 2009;72(3):80-5.

20. Kay M, Willye R. Caustic Ingestion in children. Curr Op Ped 2009;21:651-54.

21. Le Naoures P, Hamy A, Lerolle N, et al. Risk factors for symptomatic esophageal stricture after caustic ingestion-a retrospective cohort study. Diseases of the Esophagus 2017;30:1-6.

22. Mattos GM, Lopes DD, Mamede RC, et al. Effects of time of contact and concentration of caustic agent on generation of injuries. Laryngoscope 2006;116(3):456-60.

23. Khaled M. El-Asmar, Ayman M. Allam. Surgical management of corrosiveinduced gastric injury in children: 10 years' experience. J Ped Surg 2018;53:7447.

24. Salzman M, O'Malley R. Updates on the Evaluation and Management of Caustic Exposures. Emerg Med Clin N Am 2007;25459-76.

25. Mamede RC, de Mello Filho FV. Ingestion of caustic substances and its complications. Sao Paulo Med J 2001;119(1):10-5.

26. Günel E, Cağlayan F, Cağlayan O, Akillioğlu I. Reactive oxygen radical levels in caustic esophageal burns. J Pediatr Surg 1999;34(3):405-7.

27. Lamireau T, Rebouissoux L, Denis D, Lancelin F, Vergnes P, Fayon M. Accidental caustic ingestion in children: is endoscopy always mandatory?. J Pediatr Gastroenterol Nutr 2001;33:81-4.

28. Betalli P, Falchetti D, Giuliani S, et al. Caustic ingestion in children: is endoscopy always indicated? The results of an Italian multicenter observational study. Gastrointest Endosc 2008; 68:434639.

29. Boskovic A, Stankovic I. Predictability of gastroesophageal caustic injury from clinical findings: is endoscopy mandatory in children?. Eur J Gastroenterol Hepatol 26:499-503.

30. Nuutinen M, Ubari M, Kourvalainen $\mathrm{K}$. Consequences of caustic ingestion in children . Acta pediatr. 1994;83:1200-5.
31. Baskın D. A standardised protocol for the acute management of corrosive ingestion in children. Pediatr Surg Int 2004;20(1112):824-8.

32. Salzman M, O'Malley R. Updates on the Evaluation and Management of Caustic Exposures. Emerg Med Clin N Am 2007;25:459-76.

33. Temiz A1, Oguzkurt P, Ezer SS, Ince E, Hicsonmez A. Predictability of outcome of caustic ingestion by esophagogastroduodenoscopy in children. World J Gastroenterol 2012;18(10):1098103.

34. Bird JH, Kumar S, Paul C, Ramsden JD. Controversies in the management of caustic ingestion injury: an evidencebased review. Clin Otolaryngol. 2017;42(3):701-8.

35. Gorman RL, Khin-Maung-Gyi MT, KleinSchwartz W, et al. Initial symptoms as predictors of esophageal injury in alkaline corrosive ingestions. Am J Emerg Med. 1992;10:189-94.

36. Salzman M; O'Malley, R. Updates on the Evaluation and Management of Caustic Exposures. Emerg Med Clin N Am. 2007; $25: 459-76$.

37. Rodríguez Guerineau L, Martínez Sánchez L, Quintillá Martínez JM, Trenchs Sainz De La Maza V, Vila Miravet V, Luaces Cubells C. Ingesta de cáusticos: situación actual y puesta al día de las recomendaciones. Anales de Pediatría. 2011; 75(5):295-354

38. Kay M, Wyllie R. Caustic ingestions and the role of endoscopy. J Pediatr Gastroenterol Nutr 2001;32:8-10.

39. Thomson M, Tringali A, Dumonceau JM, et al. Paediatric Gastrointestinal Endoscopy: European Society for Paediatric Gastroenterology Hepatology and Nutrition and European Society of Gastrointestinal Endoscopy Guidelines. J Pediatr Gastroenterol Nutr. 2017; 64(1):133-53.

40. Uygun I, Aydogdu B, Okur MH, et al. Clinico-epidemiological study of caustic substance ingestion accidents in children in Anatolia: the DROOL score as a new prognostic tool. Acta Chir Belg 2012;112:346-54.

41. Delgado L, Acosta A, Iglesias C, et al. Esofagitis cáustica. Estenosis cáustica y su tratamiento con dilataciones. Rev Med Uru. 2006;22:46-51.

42. Kukuc G, Gollu G, Ates U, et al. Evaluation of esophageal injuries secondary to ingestion of unlabeled corrosive substances: pediatric case series. Arch Argent Pediatr. 2017;115(2):e85-8.

43. Stanley AJ, Laine L, Dalton HR, et al. Comparison of risk scoring systems for patients presenting with upper gastrointestinal bleeding: international multicentre prospective study. BMJ. 2017;356:i6432.

44. Previtera C, Giusti F, Guglielmi M. Predictive value of visible lesions (cheeks, lips, oropharynx) in suspected caustic ingestion: may endoscopy reasonably be omitted in completely negative pediatric patients? Pediatr Emerg Care. 1990;6:176.

45. Zargar SA, Kochhar R, Mehta S, Mehta SK. The role of fiberoptic endoscopy in the management of corrosive ingestion and modified endoscopic classification of burns. Gastrointest Endosc. 1991;37:165.

46. Estrera A, Taylor W, Mills LJ, Platt MR. Corrosive burns of the esophagus and stomach: a recommendation for an aggressive surgical approach. Ann Thorac Surg. 1986;41:276-83.

47. Di Costanzo J, Noirclerc M, Jouglard J, et al. New therapeutic approach to corrosive burns of the upper gastrointestinal tract. Gut 1980;21:370-5.

48. Cheng HT, Chen CL, Lin CH, et al. Caustic ingestion in adults: the role of endoscopic classification in predicting outcome. BMC Gastroenterology 2008;8:31.

49. Shub MD. Therapy of caustic ingestion: new treatment considerations. Curr Opin Pediatr 2015;27:609-13.

50. Arnold M, Numanoglu A. Caustic ingestion in children - A review. Seminars in Pediatric Surgery 2017;26:95-104.

51. Lightdale JR, Acosta R, Shergill AK, et al. Modifications in endoscopic practice for pediatric patients. Gastr Endosc 2014;79(5):699-710.

52. De Jong AL, Macdonald R, Ein S, Forte $\mathrm{V}$, Turner A. Corrosive esophagitis in children: a 30-year review. Int J Pediatr Otorhinolaryngol. 2001;57:203-11.

53. Millar A, Numanoglu A, Mann M, et al. Detection of caustic oesophageal injury with technetium $99 \mathrm{~m}$-labelled sucralfate. J Pediatr Surg. 2001;36(2):262-5.

54. Kamijo Y, Kondo I, Kokuto M et al. Miniprobe Ultrasonography for Determining Prognosis in Corrosive Esophagitis. Am J Gastroenterol. 2004; 99(5):851-85.

55. Chiu HM, Lin JT, Huang SP, et al. Prediction of bleeding and stricture formation after corrosive ingestion by EU concurrent whith upper endoscopy. Gastrointest Endosc 2004;60(5):827-83.

56. Bonicci KS, Wood DW, Dargan PI. Should computerized tomography replace endoscopy evaluation of symptomatic ingestion of corrosive substances?. Clin Toxicol 2014;52:911-25.

57. Chirica M, Resche-Rigon M, Pariente B, et al. Computed tomography evaluation of high-grade esophageal necrosis alter corrosive ingestion to avoid unnecessary esophagectomy. Surg Endosc 2015;29:1452-61. 